\title{
Preventing invasive Group B Streptococcus (GBS) disease in South African infants: Time for change
}

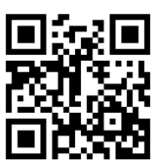

Streptococcus agalactiae is an encapsulated Gram-positive coccus that colonises the gastrointestinal and genitourinary tracts. This organism belongs exclusively to Group B in Lancefield's grouping of Streptococcus species and therefore is referred to as Group B Streptococcus (GBS). Of the ten known serotypes, serotypes Ia and III account for most disease. The greatest burden of invasive GBS disease is seen in infants $<3$ months of age, although GBS can cause disease in adults and pregnant women. Disease manifesting in the first 6 days of life is referred to as early-onset disease (EOD), whereas late-onset disease (LOD) manifests from day 7 - 89 of life.

Approximately 10 - 40\% of pregnant women are colonised, rectally and/or vaginally, with GBS. About $30-70 \%$ of newborns become colonised with GBS in utero or during delivery, and invasive disease occurs in 1 - 3\% (probably from aspiration of infected amniotic fluid or genital secretions). ${ }^{[1]}$ Most EOD (>90\%) manifests at birth, with signs of respiratory distress or sepsis. Furthermore, there is a strong association between GBS disease and stillbirth. ${ }^{[2]}$ In contrast, LOD occurs through horizontal transmission via the faecal-oral route (the mother is usually the source), through infected breastmilk, or rarely, through nosocomial spread. These infants may develop sepsis, meningitis ( 50\%), septic arthritis, osteomyelitis or cellulitis.

The global incidence (per 1000 live births) of invasive GBS disease in infants $<90$ days of age is estimated to be 0.53 ( $95 \%$ confidence interval (CI) $0.44-0.62$ ) with a case fatality ratio of $9.6 \% \cdot{ }^{[3]}$ In South Africa (SA), the high incidence rate has remained largely unchanged at $2-3$ per 1000 live births over the last two and half decades, ${ }^{[4-7]}$ although it is reportedly lower in the Western Cape (0.67 per 1000 live births). ${ }^{[8]}$ Of concern, the high incidence is also associated with a high case fatality ratio (13 - 18\%) and there is significant neurological impairment among survivors, especially in those with meningitis (24\%). ${ }^{[7]}$

Worldwide, the highest incidence of invasive GBS disease is reported in SA: $;{ }^{[9]}$ this is likely to contribute to the high neonatal mortality rate and our subsequent failure to reach the targeted goal of reducing under-five childhood mortality (Millenium Development Goal 4). Trends in the incidence of invasive GBS disease in Soweto from 1997 to 2013 have highlighted that suboptimal implementation of intrapartum antibiotic prophylaxis (IAP) to women identified to be at risk of having infants with EOD, coupled with maternal HIV infection being associated with a two-to-five-fold greater risk of invasive GBS disease in infants, has contributed to the country persistently reporting the high rates of invasive GBS disease. ${ }^{[6,7]}$ The increased susceptibility to invasive GBS disease in infants born to HIV-infected women is thought to be due to lower maternal protective GBS capsular antibody concentrations and the inefficient transplacental transfer thereof. ${ }^{[10]}$

In the USA, the incidence of EOD has declined significantly $(>80 \%)$ since the early 1990s through effective execution of secondary preventative strategies, i.e. antibiotics administered intravenously 4 hours before delivery to GBS-colonised women who were screened at $35-37$ weeks' gestation. ${ }^{[1]}$ This universal screening approach (as recommended by the Centers for Disease Control (CDC)) cannot be readily implemented in most low-to-middleincome countries, where a large proportion of deliveries occur outside health facilities, although this is not the case in SA. In addition, the high cost of screening pregnant women, providing IAP and creating the necessary infrastructure that enables the administration of intravenous IAP at least 4 hours prior to delivery has hampered preventive efforts in low-to-middleincome countries. Furthermore, the CDC recommendation has limitations such as false-negative cultures in colonised pregnant women, failure to institute IAP for sudden deliveries, the risk of penicillin resistance and the limited effect of IAP on preventing preterm delivery, stillbirths and LOD. ${ }^{[1]}$

Currently, most low-to-middle-income counties, including SA and some European countries, practise the less successful riskbased approach to prevent invasive disease, i.e. IAP is only provided to mothers in labour with risk factors such as maternal fever, prolonged rupture of membranes or prematurity. This strategy is also failing in low-to-middle-income countries because risk factors do not identify all women who are at risk of transmitting GBS. Furthermore, healthcare workers may fail to recognise risk factors, and may not provide IAP in busy obstetric units. Other proposed preventive strategies, including chlorhexidine vaginal wipes, do not prevent EOD. Rapid molecular diagnostic tests to detect maternal GBS colonisation during labour is promising but costly, and the struggle for most low-tomiddle-income countries remains the timely and effective administration of IAP, thus rendering molecular tests futile in these settings.

The above limitations, as well as the failure to implement these strategies in low-to-middle-income countries, calls for a paradigm shift regarding the future prevention of invasive GBS disease. Vaccinating pregnant women in the second trimester of pregnancy could result in an increase in maternal antibody levels, which in turn may be transferred to the fetus in the last trimester. This strategy

Table 1. Antibiotic therapy in infants with invasive GBS disease

\begin{tabular}{llll}
\hline Clinical presentation & $\begin{array}{l}\text { Penicillin G } \\
\text { (units/kg per day) }\end{array}$ & $\begin{array}{l}\text { Ampicillin }(\mathrm{mg} / \\
\text { kg per day) }\end{array}$ & $\begin{array}{l}\text { Duration of } \\
\text { antibiotics (days) }\end{array}$ \\
\hline Bacteraemia without a focus & $75000-150000$ & $100-200$ & $10-14$ \\
Sepsis & $75000-150000$ & $100-200$ & $10-14$ \\
Pneumonia & $75000-150000$ & $100-200$ & $10-14$ \\
Urinary tract infection & $75000-150000$ & $100-200$ & $10-14$ \\
Cellulitis & $75000-150000$ & $100-200$ & $10-14$ \\
Septic arthritis & $75000-150000$ & $100-200$ & $14-21$ \\
Osteomyelitis & $75000-150000$ & $100-200$ & $21-28$ \\
Meningitis & $450000-500000$ & $200-300$ & 21
\end{tabular}

*The recommended dosing interval for Penicillin G is 8 or 12 hourly, except for meningitis, for which the recommendation is 6 hourly. For early-onset meningitis, the dose of Penicillin G is 250000 - 450000 units $/ \mathrm{kg}$ per day divided every 8 hours for the first week of life. The recommended dosing interval for ampicillin is 6 hourly, except during the first week of life where the recommendation is 8 or 12 hourly. Gentamicin $5 \mathrm{mg} / \mathrm{kg} /$ day for 5 days can be used for synergy in cases of meningitis. 
has been successfully used for the prevention of tetanus, influenza and pertussis in infants. ${ }^{[11]} \mathrm{A}$ trivalent GBS polysaccharide-protein conjugate vaccine (against serotypes Ia, Ib and III) has completed phase-II evaluation among pregnant women and has the potential to prevent $70-80 \%$ of all invasive GBS disease. Maternal GBS vaccination is potentially cost effective and easy to implement in low-to-middle-income settings; furthermore, vaccination during pregnancy is becoming more acceptable to the public.

For those infants who require treatment for invasive GBS disease, penicillin remains the drug of choice (Table 1). There is no role for the use of immunoglobulins or dexamethasone in infants with meningitis. Recurrence of invasive GBS disease may occur in 1 - 2\% of infants despite adequate treatment.

In conclusion, GBS is the most common cause of neonatal sepsis and meningitis. SA has a very high incidence of invasive GBS disease and the provision of IAP is practically difficult; maternal GBS vaccination is a promising strategy.

\section{Z Dangor, ${ }^{1,2,3}$ S G Lala, ${ }^{1}$ S A Madhi ${ }^{2,3,4}$}

${ }^{1}$ Department of Paediatrics and Child Health, Faculty of Health Sciences, University of the Witwatersrand, Johannesburg, South Africa ${ }^{2}$ Medical Research Council: Respiratory and Meningeal Pathogens Research Unit, University of the Witwatersrand, Johannesburg, South Africa

${ }^{3}$ Department of Science and Technology/National Research Foundation: Vaccine Preventable Diseases, University of the Witwatersrand, Johannesburg, South Africa

${ }^{4}$ National Institute for Communicable Diseases: A division of National Health Laboratory Service, Johannesburg, South Africa

Financial disclosure/support. ZD is funded in part by the Carnegie Corporation of New York (Grant number B8749) and the Discovery Foundation (Grant number 20289/1). SAM is funded in part by National Research Foundation/Department of Science and Technology: South African Research Chair Initiative in Vaccine Preventable Diseases and
Medical Research Council of South Africa. The funders had no role in study design, data collection and analysis, decision to publish, or preparation of the manuscript.

\section{Corresponding author: Z Dangor (ziyaad.dangor@wits.ac.za)}

1. Verani JR, McGee L, Schrag SJ. Prevention of perinatal group B streptococcal disease: Revised guidelines from CDC, 2010. Mor Mortal Wkly Rep 2010;59(RR-10):1-36.

2. Nan C, Dangor Z, Cutland CL, Edwards MS, Madhi SA, Cunnington MC. Maternal group B Streptococcus-related stillbirth: A systematic review. BJOG 2015 (in press). [http://dx.doi.org/10.1111/1471-0528.13527]

3. Edmond KM, Kortsalioudaki C, Scott S, et al. Group B streptococcal disease in infants aged younger than 3 months: Systematic review and metaanalysis. Lancet 2012;379(9815):547-556. [http://dx.doi.org/10.1016/S01406736(11)61651-6]

4. Haffejee IE, Bhana RH, Coovadia YM, Hoosen AA, Marajh AV, Gouws E. Neonatal group B streptococcal infections in Indian (Asian) babies in South Africa. J Infect 1991;22(3):225-231.

5. Madhi SA, Radebe K, Crewe-Brown $\mathrm{H}$, et al. High burden of invasive Streptococcus agalactiae disease in South African infants. Ann Trop Paediatr 2003;23(1):15-23. [http://dx.doi.org/10.1179/000349803125002814]

6. Cutland CL, Schrag SJ, Thigpen MC, et al. Increased risk for Group B Streptococcus sepsis in young infants exposed to HIV, Soweto, South Africa, 2004 - 2008. Emerg Infect Dis 2015;21(4):638-645.

7. Dangor Z, Lala SG, Cutland CL, et al. Burden of invasive Group B Streptococcus disease and early neurological sequelae in South African infants. PloS ONE 2015;10(4):e0123014. [http://dx.doi.org/10.1371/journal.pone.0123014]

8. Frigati L, van der Merwe JL, Harvey J, Rabie H, Theron G, Cotton MF. A retrospective review of Group B streptococcal infection in the Metro East area of the Western Cape province: 2010 - 2011. S Afr J Infect Dis 2014;29(1):33-36.

9. Dagnew AF, Cunnington MC, Dube Q, et al. Variation in reported neonatal group B streptococcal disease incidence in developing countries. Clin Infect Dis 2012;55(1):91-102. [http://dx.doi.org/10.1093/cid/cis395]

10. Dangor Z, Kwatra G, Izu A, et al. HIV-1 is associated with lower Group B Streptococcus capsular and surface-protein IgG antibody levels and reduced transplacental antibody transfer in pregnant women. J Infect Dis 2015;212(3):453-462. [http://dx.doi.org/10.1093/infdis/jiv064]

11. Steinhoff MC. Assessments of vaccines for prenatal immunization. Vaccine 2013;31(Suppl 4):D27-30. [http://dx.doi.org/10.1016/j.vaccine.2013.02.031]

S Afr J Child Health 2015;9(3):69-70. DOI:10.7196/SAJCH.8575 